\title{
Effects of temperature variations on guided waves propagating in composite structures
}

\author{
Siavash Shoja*, Viktor Berbyuk, Anders Boström \\ Dept. of Applied Mechanics, Chalmers University of Technology, SE-412 96, Gothenburg, Sweden
}

\begin{abstract}
Effects of temperature on guided waves propagating in composite materials is a well-known problem which has been investigated in many studies. The majority of the studies is focused on effects of high temperature. Understanding the effects of low temperature has major importance in composite structures and components which are operating in cold climate conditions such as e.g. wind turbines operating in cold climate regions. In this study first the effects of temperature variations on guided waves propagating in a composite plate is investigated experimentally in a cold climate chamber. The material is a common material used to manufacture rotor blades of wind turbines. The temperature range is $25^{\circ} \mathrm{C}$ to $-25^{\circ} \mathrm{C}$ and effects of temperature variations on amplitude and phase shift of the received signal are investigated. In order to apply the effects of lowering the temperature on the received signal, the Baseline Signal Stretch (BSS) method is modified and used. The modification is based on decomposing the signal into symmetric and asymmetric modes and applying two different stretch factors on each of them. Finally the results obtained based on the new method is compared with the results of application of BSS with one stretch factor and experimental measurements. Comparisons show that an improvement is obtained using the BSS with the mode decomposition method at temperature variations of more than $25^{\circ} \mathrm{C}$.
\end{abstract}

\section{INTRODUCTION}

Guided waves are one efficient tool in fields of inspection, detection and non-destructive evaluation when it comes to elongated structures. The main property of this kind of waves, which makes them suitable for many applications, is the ability to propagate along long distances without significant loss of energy, thus covering large areas with limited number of sensors. Although guided waves are an accurate tool for Structural Health Monitoring (SHM), they can be easily affected by environmental conditions. Since temperature is an important environmental condition, studying how temperature changes alter the recorded waveform has attracted interest [1]. One example is wind turbines which operate at high altitudes or in cold regions confronting a $+30^{\circ} \mathrm{C}$ to $-30^{\circ} \mathrm{C}$ temperature change [2].

Previous studies have been shown that a change of temperature is one of the main sources of fluctuations in the received signal [3]. This effect is due to various parameters from thermal expansion of the material, change in stiffness of the material together with the effect of temperature change on piezoelectric materials including transducers and sensors and their bonds [4]. Some methodologies have been introduced in order to reduce the effects of temperature variation, however, it has been said that the effects cannot be entirely suppressed [3-5]. It is possible by a careful selection of the adhesive for bonding the transducers and the transducers themselves to minimize the effect of temperature on them. However, the changes in the material properties, including expansion and changing the stiffness of the materials, are not possible to manipulate. The main effects of temperature change on the material which is of interest here are changes in the elastic moduli and the density of the material. Previous studies $[6,7]$ show that these effects can be divided into two smaller effects:

1. Changes in phase of the received signal.

2. Changes in amplitude of the received signal.

The change in phase of the received signal is a consequence of changing the velocity of the wave. Due to thermal expansion in the material, both group and phase velocities of the wave change. In general, it is possible to say that lower temperatures

*siavash.shoja@chalmers.se; phone +46 31772 3476; www.chalmers.se/am

Smart Materials and Nondestructive Evaluation for Energy Systems 2016, edited by

Norbert G. Meyendorf, Theodoros E. Matikas, Kara J. Peters, Proc. of SPIE Vol. 9806, 980605

· (c) 2016 SPIE · CCC code: 0277-786X/16/\$18 - doi: 10.1117/12.2218791 
increase the elastic moduli of the composite material and decrease the damping properties [8]. These changes cause the velocity of the wave to increase by lowering the temperature and vice versa. Due to the orthotropic characteristics of composite materials, the effects of temperature can be different in different directions.

Many investigations have been performed in order to introduce a method to predict the effects of temperature on the received signal. Two of these methods are Optimal Baseline Selection (OBS) and Baseline Signal Stretch (BSS), which both are developed based on signal processing fundamentals $[1,5]$. The methodology of OBS uses multiple baseline measurements recorded over a range of temperatures whereas the BSS method is based on stretching the signal and altering the amplitude. Croxford et al. [6] performed a study on both methods and concluded that the combination of OBS and BSS is a robust practical solution to temperature compensation. Although many studies have been devoted to this topic, there are few that focus on composite components. In some investigations a linear dependency of properties of the composite material is assumed in order to vary them by changing the temperature [9, 10]. Park and Jun [11] applied the BSS method to consider temperature variations in a composite plate by considering a linear dependency for the stretch factor and amplitude change.

The purpose of this study is first to show and discuss the effects of temperature changes on guided waves propagating along a glass-fiber composite plate using an experimental setup. Then it is demonstrated that it is possible to improve the results by modifying the BSS method considering mode decomposition and applying two stretch factors. Most of the studies on this topic are done on high temperatures and since low temperatures can have different possible effects on the transducers and sensors, this study is more focused towards this.

\subsection{Experimental setup}

\section{EXPERIMENTAL STUDY}

The plate used in this study is a glass-fiber composite plate containing 62 layups and Vinylester resin, a common material used in the wind turbine industry. The plate is designed in a way that about $56 \%$ of the layups are unidirectional in the longitudinal direction and $30 \%$ of them are in $45^{\circ}$. The dimensions of the plate are $0.02 \times 0.2 \times 8 \mathrm{~m}$. The plate is excited by means of a magnetostrictive actuator using Terfenol-D with $5^{\circ}$ angle of inclination. Details about the design of the transducer is fully described in previous work [12]. To measure the acceleration of the displacements of the propagating wave, piezoelectric accelerometers of the type IMI608A11 are used with coaxial cables RG58. 24 accelerometers are mounted inside aluminum cubes and glued equally spaced on the surface of the plate between $2 \mathrm{~m}$ and $6 \mathrm{~m}$ from the excitation side. The accelerometers are mounted in such a way that they mostly measure the signal in the longitudinal direction. The output of the accelerometers is an electric signal that is sent to an NI data acquisition system for post processing (Figs. 1 and 2).

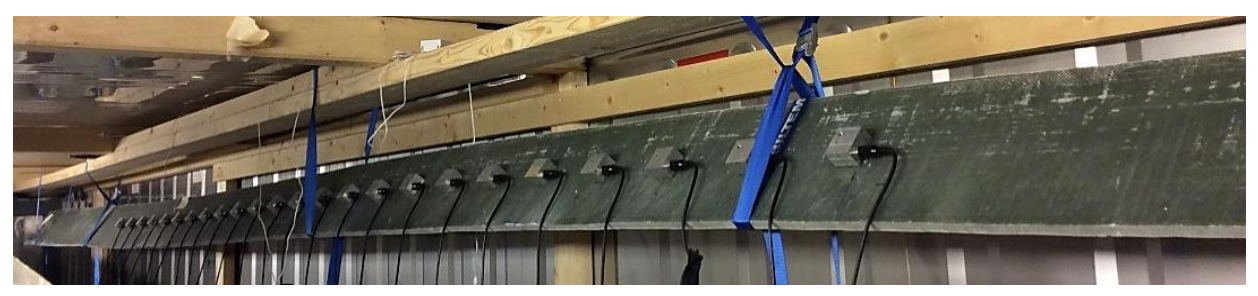

Figure 1. The composite test object with the accelerometers mounted on it in the cold climate lab. 


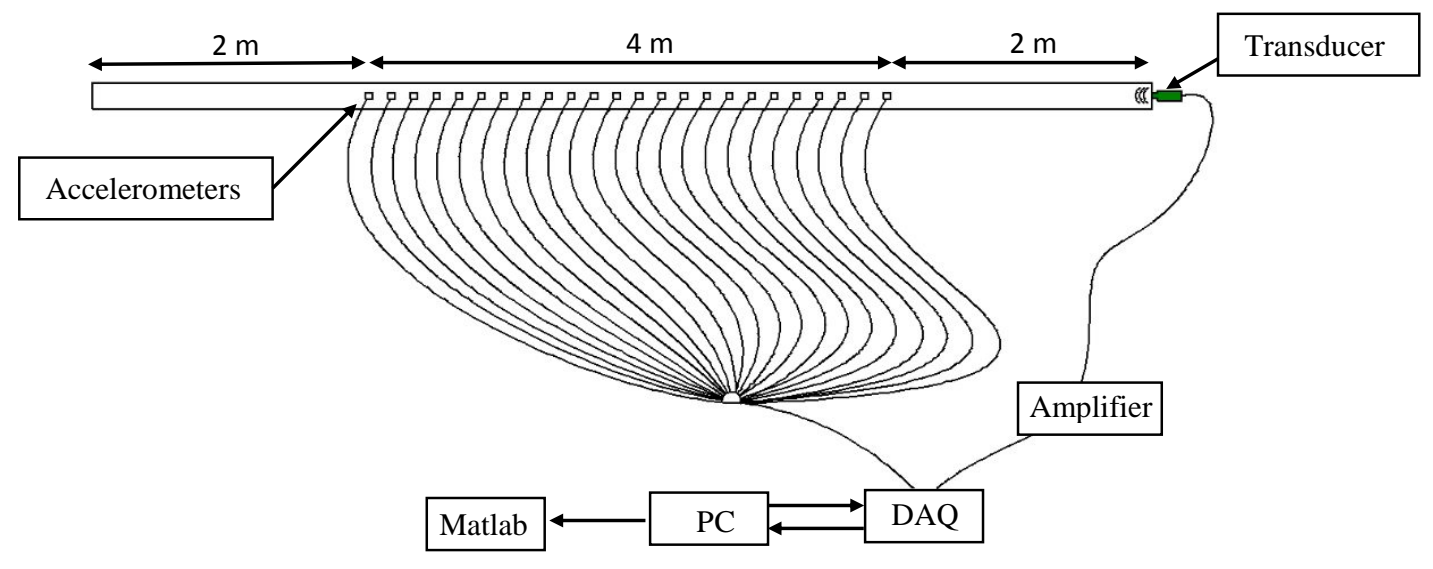

Figure 2. Schematic view of the experimental setup.

The excitation signal is a windowed sinus signal with a center frequency of $4 \mathrm{kHz}$. This kind of signal is widely used in guided-wave applications because it gives good signal strength and avoids dispersion over long propagation distances. Moreover, using this signal it is possible to ensure that a limited part of the frequency spectrum is always excited (Fig. 3) [13].
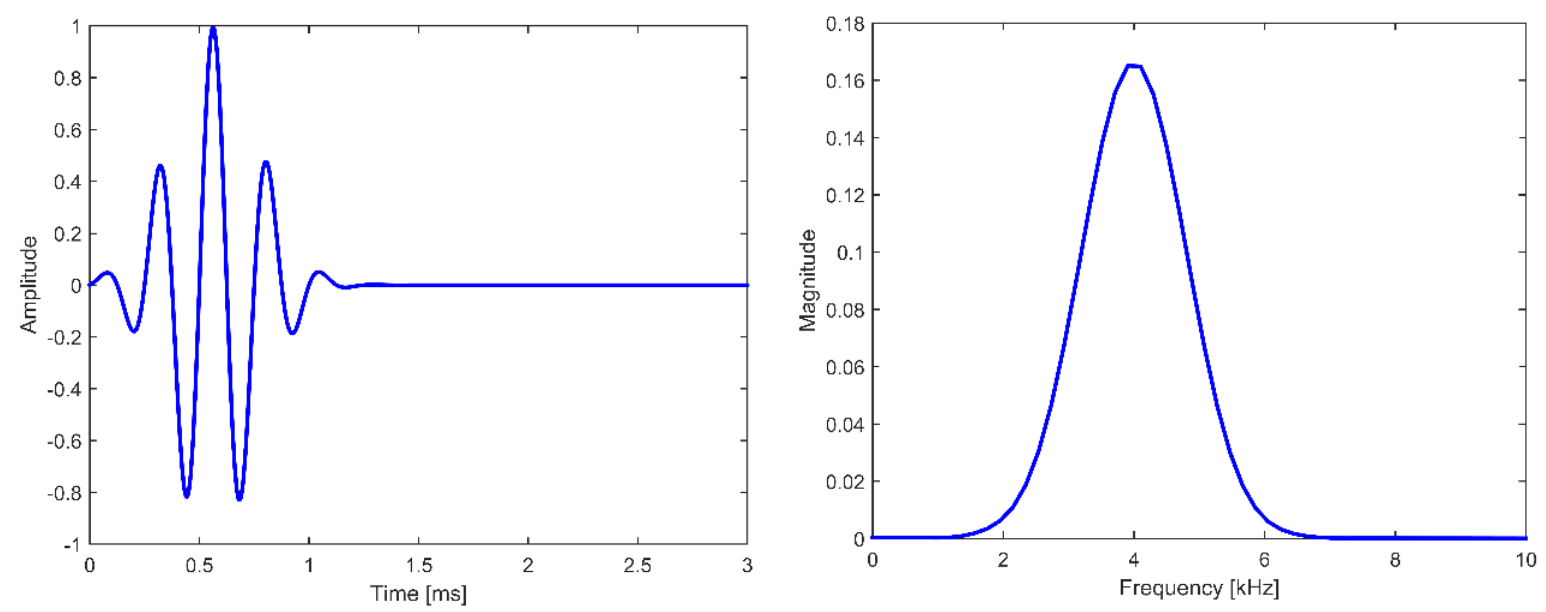

Figure 3. The excitation signal in the time domain (left) and the frequency domain (right).

The experimental work is done in a chamber, designed for cold climate studies. Using this chamber, it is possible to change the surrounding temperature from $+25^{\circ} \mathrm{C}$ to $-25^{\circ} \mathrm{C}$. Two thermometers are used to measure the temperature, one for the surrounding temperature and one glued to the test object to measure its surface temperature.

\subsection{Measurement and data analysis}

Since the frequency of the noise is close to the excitation frequency, it is challenging to filter the noise using common filtering methods. Here each experiment is run 9 times and the average value of all the time signals were calculated and considered as the received signal. Using this method, the amplitude of noise is decreased about $30 \%$. The received signal in the frequency domain is filtered using a Gaussian filter according to the following

$$
U_{x, F}(f)=U_{x}(f) \mathrm{e}^{-K\left(f-f_{n}\right)^{2}},
$$

where $K$ is the coefficient which defines the bandwidth of the filter and $f_{n}$ is the central frequency of the filter. The signal is generated in the time domain after filtering using an inverse Fourier transform. 

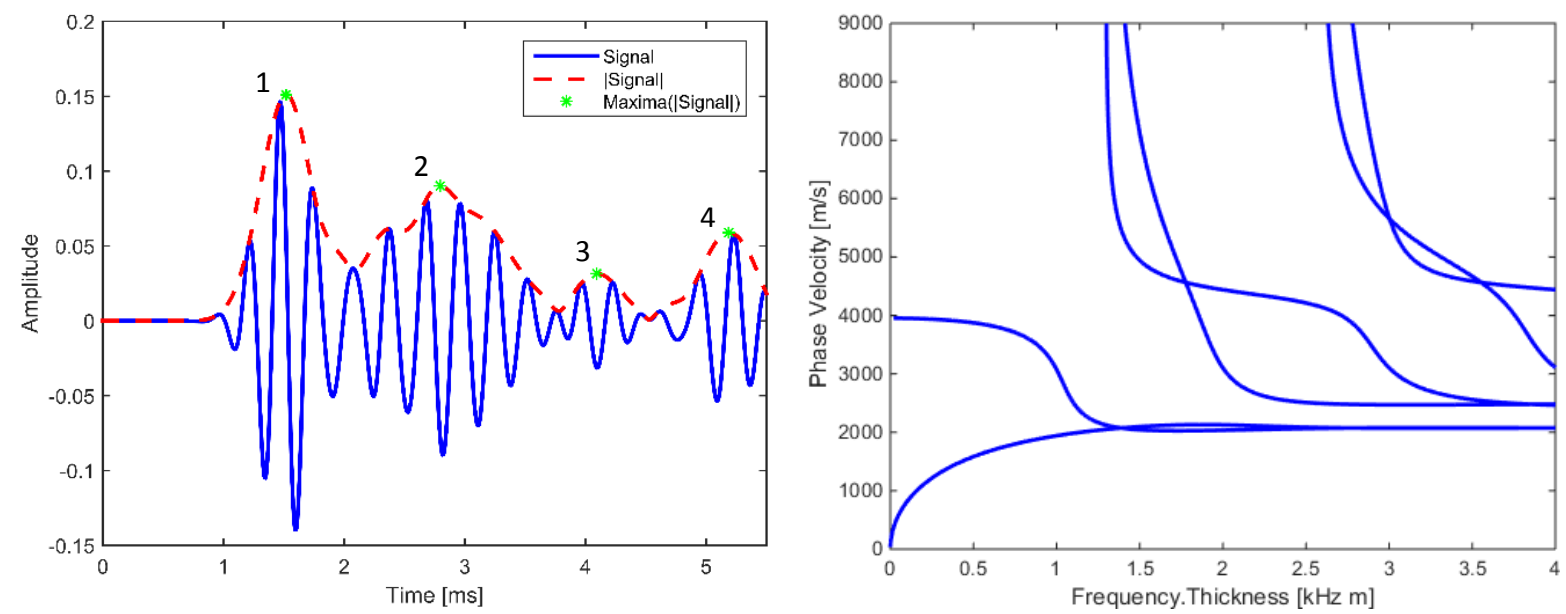

Figure 4. The received waveform from the 5 th accelerometer (left) and dispersion curves calculated for the composite plate in a $2 \mathrm{D}$ domain (right).

Figure 4 (left) illustrates the time signal received at the 5th accelerometer when the plate is excited with $4 \mathrm{kHz}$ center frequency at $25^{\circ} \mathrm{C}$. In order to find the wave packets, the absolute value of the time signal is calculated. By finding the local maximum points (peaks) of the absolute value of the signal, it is possible to find the wave packets.

In order to understand the time signal, dispersion curves of the current material have been calculated and illustrated in Fig. 4 (right). Homogenization of the composite material to calculate the dispersion curves is done using Classical Laminate Theory and First-order Shear Deformation Theory [2]. The dispersion curves show that one symmetric and one asymmetric mode are expected at this frequency (approximately $0.08 \mathrm{kHz} . \mathrm{m}$ ). However, three wave packets are measured in the experimental work which each introduce a wave mode (the fourth wave packet is the reflection from the far end of the plate). Since the dispersion curves are calculated in a 2D domain and the width of the plate is assumed infinite, the third mode can be due to the limited width of the plate in the experiment.

Previous studies show that due to the special characteristics of the piezoelectric material in the transducers and accelerometers together with the effect of temperature on dielectric permittivity terms, they show different behavior in different temperature intervals [9]. Lanza di Scalea and Salamone [14] investigated the temperature effect on isotropic materials using ultrasonic Lamb waves and they showed that the amplitude decreases by increasing the temperature between $+20^{\circ} \mathrm{C} \leq T \leq+60^{\circ} \mathrm{C}$ and it increases by increasing the temperature between $-40^{\circ} \mathrm{C} \leq T \leq+20^{\circ} \mathrm{C}$.

In the current work, the temperature of the chamber is changed between $+25^{\circ} \mathrm{C}$ and $-25^{\circ} \mathrm{C}$ and the received signals are compared (Fig. 5). It can be observed that the amplitude of the signals diminishes for both symmetric and asymmetric modes which is according to the predictions. 


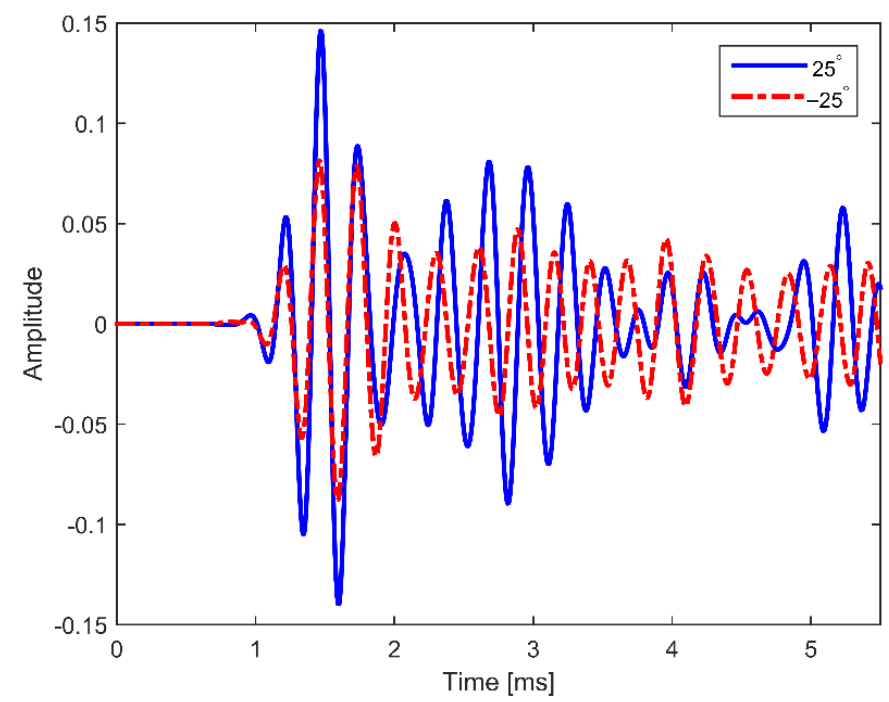

Figure 5. A comparison on the received signals at $25^{\circ} \mathrm{C}$ and $-25^{\circ} \mathrm{C}$.

For better understanding, maximum values of the amplitudes of the received signals are measured for both symmetric and asymmetric modes at several temperatures as illustrated in Fig. 6. The graph shows that the maximum amplitude for both of the modes increases by increasing the temperature, however, the rise happens with two different rates. By fitting a linear curve to the graphs of maximum amplitude and comparing the slopes, it is observed that the amplitude of the symmetric mode increases $75 \%$ faster compared to the asymmetric mode.

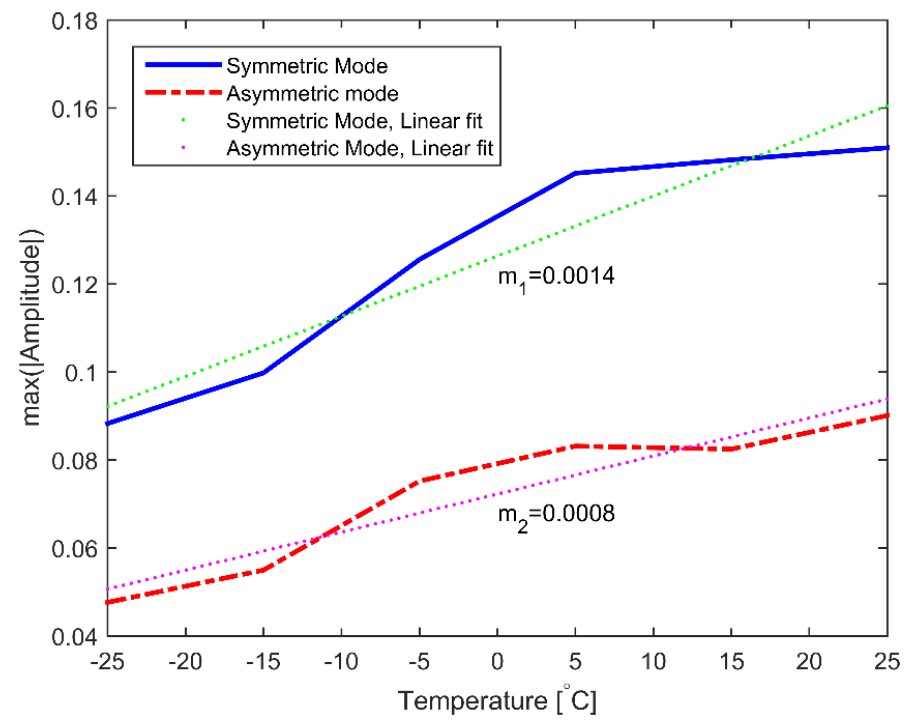

Figure 6. A comparison between the maximum amplitude of the signal for two different wave modes.

To observe the effect of temperature variations on the velocity of the wave, the time lag between temperature intervals has been calculated individually for symmetric and asymmetric modes. Since the wave packets are determined previously, it is possible to separate them by applying a Hanning window function. The average value of the time lag between every two signals in temperature intervals are calculate using a cross-correlation method [15]. Figure 7 illustrates the time delay for both symmetric and asymmetric modes in every temperature interval. By fitting a linear curve to the graphs and comparing the slops of the curves, it is concluded that the time delay in asymmetric modes increases five times faster compared to the symmetric modes. 


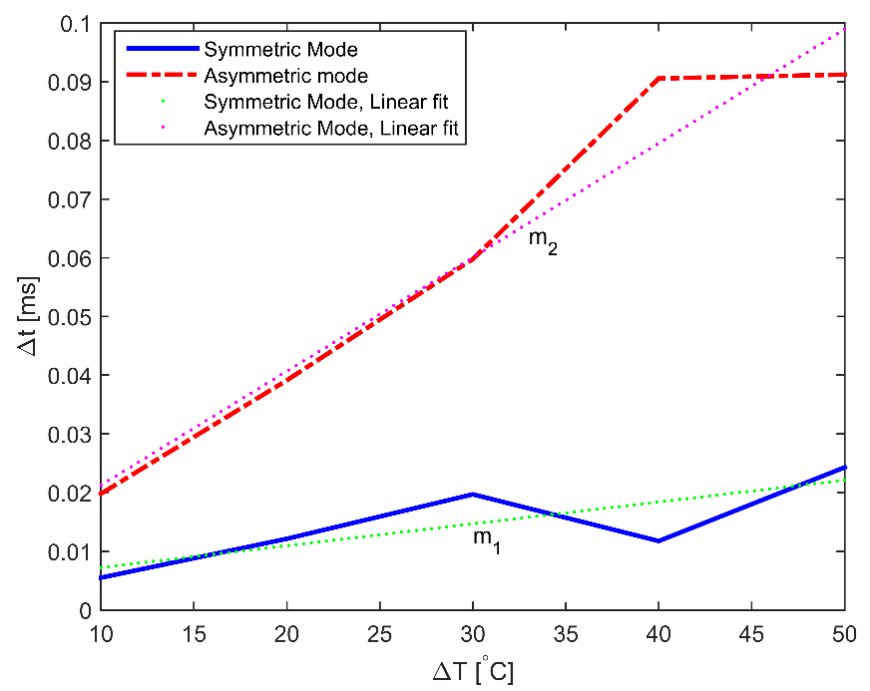

Figure 7. A comparison between the time lag of the signal for two different wave modes versus temperature change.

One way to explain this phenomenon is since about $60 \%$ of the fibers are in the longitudinal direction (both UDs and $45^{\circ} \mathrm{s}$ influence the material characteristics in the longitudinal direction), there is a large difference between coefficients of thermal expansion in the longitudinal and transverse direction.

Studying the effect of temperature variation shows that both amplitude and group velocity of the guided waves change with different rate for symmetric and asymmetric modes.

\section{BASELINE SIGNAL STRETCH}

One method for temperature compensation is the BSS method. In this method, a signal in the time domain is modified in such a way that it matches to another signal. In this part of the study, first this method is explained, then it is modified in a way that is useful for guided wave propagation in composite materials.

\subsection{The basics of the method}

A recorded time signal from a guided wave propagating in a medium at a specific temperature is assumed to be a sum of several wave packets with different wave forms and amplitudes. This can be written as

$$
u(t)=\sum_{j=1}^{N} A_{j} S_{j}\left(t-t_{j}\right),
$$

where $t$ is time, $A_{j}, S_{j}$ and $t_{j}$ are respectively the amplitude, wave-form and arrival time of the $j$ :th wave packet. In the BSS method the time axis of the time signal $(u(t))$ is stretched by a stretch factor $\alpha$ which is a function of temperature. The new time signal can be written as

$$
v(t, \alpha)=\sum_{j=1}^{N} A_{j} S_{j}\left(\alpha t-t_{j}\right) .
$$

It is worth to mention that the term stretch refers to both stretch and compression depending on the value of the stretch factor. 
In order to implement the stretch factor an approach is used which is based on calculating the FFT of the signal. So the stretch factor is applied in FFT calculations:

$$
X_{f}=C \sum_{t=1}^{N} u(t) e^{\frac{-2 \pi i}{N}(t-1)(f-1) \alpha}
$$

where

$$
C=e^{\frac{-2 \pi i}{N}(\alpha-1)(f-1)} .
$$

Now by computing the inverse FFT of $X_{f}$, it is possible to build the stretched time signal $(v(t, \alpha))$ with a stretch factor $\alpha$.

\subsection{Implementing BSS with Mode Decomposition (MD)}

As mentioned for composite materials which have orthotropic characteristics, the effect of temperature applies differently on symmetric and asymmetric wave modes. Therefore applying the BSS method with one stretch factor does not seem an appropriate approach. So it is proposed to apply two different stretch factors, one for each wave mode. In this case, it is possible to represent the time signal

$$
u(t)=\sum_{i=1}^{N} A_{i}^{s} S_{i}^{S}\left(t-t_{i}\right)+\sum_{j=1}^{M} A_{j}^{a} S_{j}^{a}\left(t-t_{j}\right),
$$

in the form of

$$
v(t, \alpha, \beta)=\sum_{i=1}^{N} A_{i}^{s} S_{i}^{s}\left(\alpha t-t_{i}\right)+\sum_{j=1}^{M} A_{j}^{a} S_{j}^{a}\left(\beta t-t_{j}\right) .
$$

Here $A_{i}^{s}, S_{i}^{s}, A_{j}^{a}, S_{j}^{a}, \alpha$ and $\beta$ are respectively amplitude, wave form and stretch factor of the symmetric and asymmetric wave modes. The stretch factors are calculated using a nonlinear least-squares data fitting between the analytical method and the experimental measurements where the sum of squares of the residuals is minimized.

Since different stretch factors are applied on the signal, noise will be created at the point where the two wave packets are connected. This noise is reduced later by applying a Gaussian filter.

One of the major assumptions of the modified BSS method is the ability of decomposing the signal into different wave modes. Since in this study the signal is recorded for a short period of time, it is possible to decompose the signal by looking at the wave packets. However, this becomes more challenging when a longer period of time is recorded and more reflections are created. Park et al. [16] introduced a method by comparing the amplitude and group velocity of each wave packet to recognize the wave modes which can be used in larger time spans.

\subsection{Comparison with experiment}

In order to examine the accuracy of the proposed method, it is applied on the experimental measurements at $25^{\circ} \mathrm{C}$ using stretch factors for a specific temperature lower than $25^{\circ} \mathrm{C}$ and comparing with the experimental measurements at this temperature. As explained before, the stretch factor for each wave mode at a temperature lower than $25^{\circ} \mathrm{C}$ is calculated by running a data fitting method. Figure 8 shows the calculated factors for each temperature interval. 


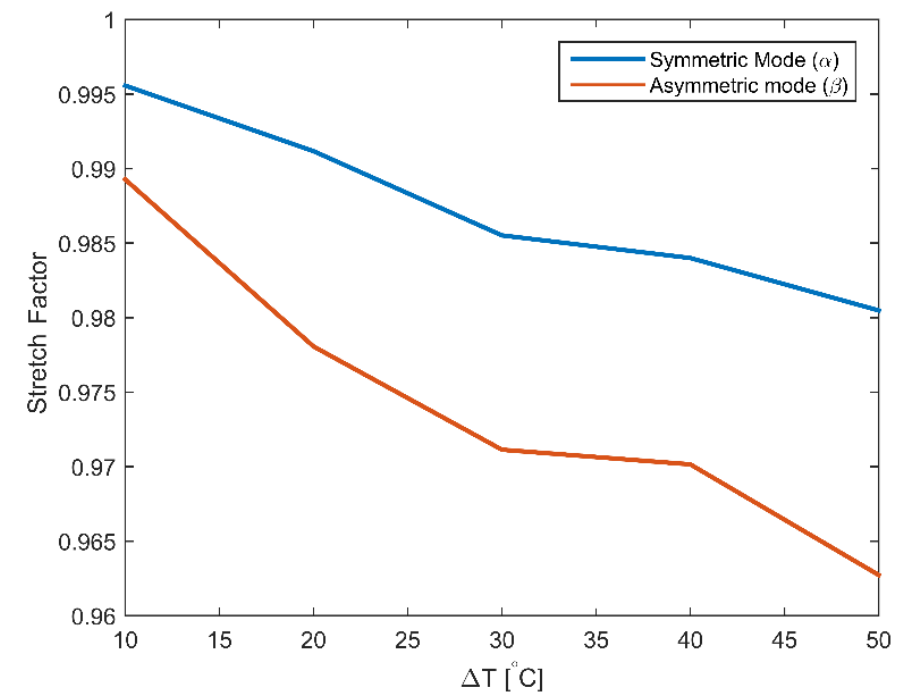

Figure 8. A comparison between stretch factors calculated for both symmetric and asymmetric modes in a range of temperature intervals.

Moreover the BSS method with MD is also compared with the BSS method with one stretch factor to understand how much this can improve the results. Figures 9 (a-e) show the comparison made on the time signal at different temperatures from $15^{\circ} \mathrm{C}$ to $-25^{\circ} \mathrm{C}$.

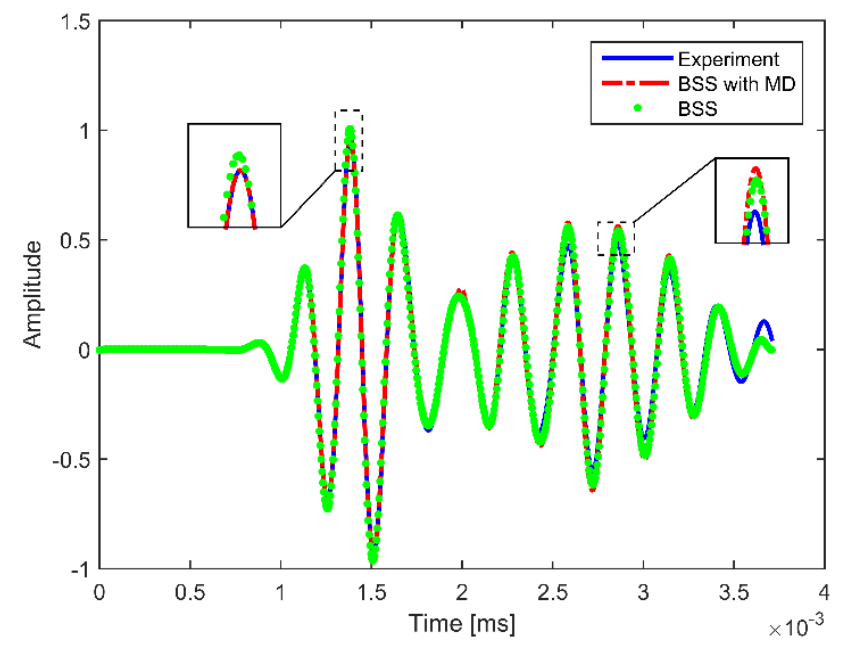

(a)

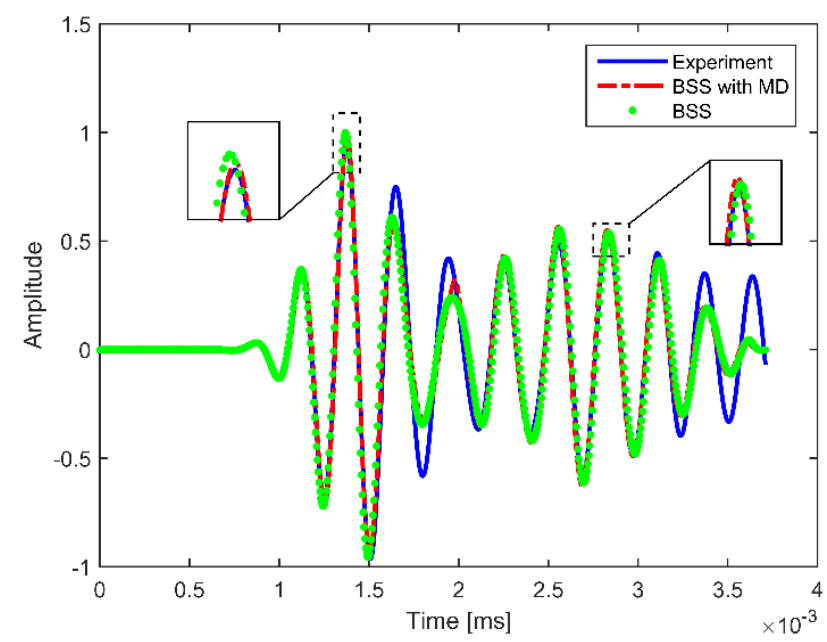

(b) 


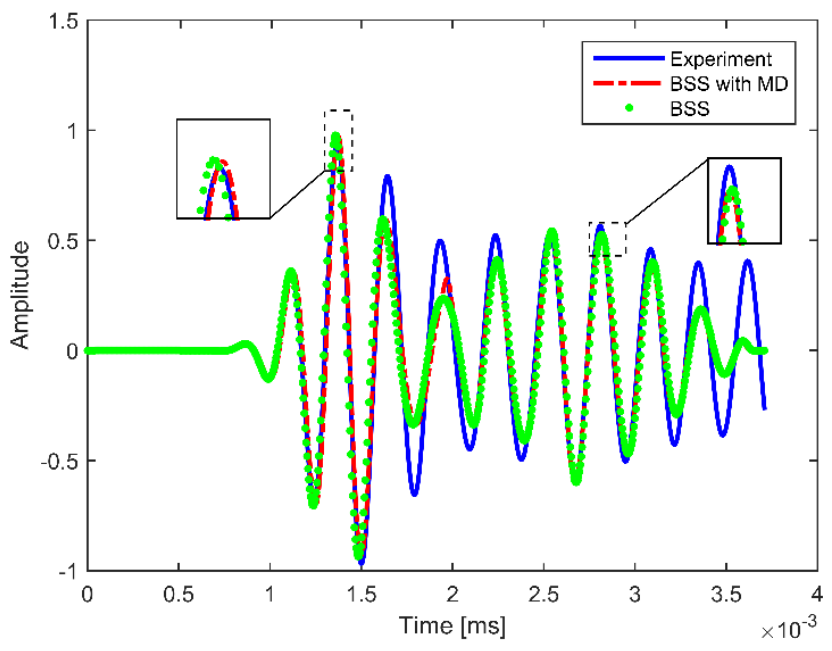

(c)

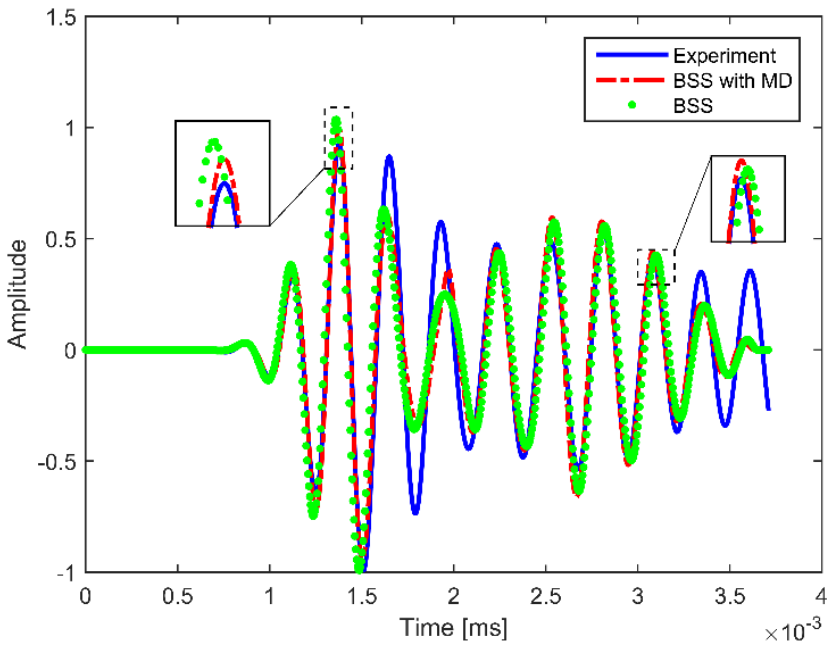

(d)

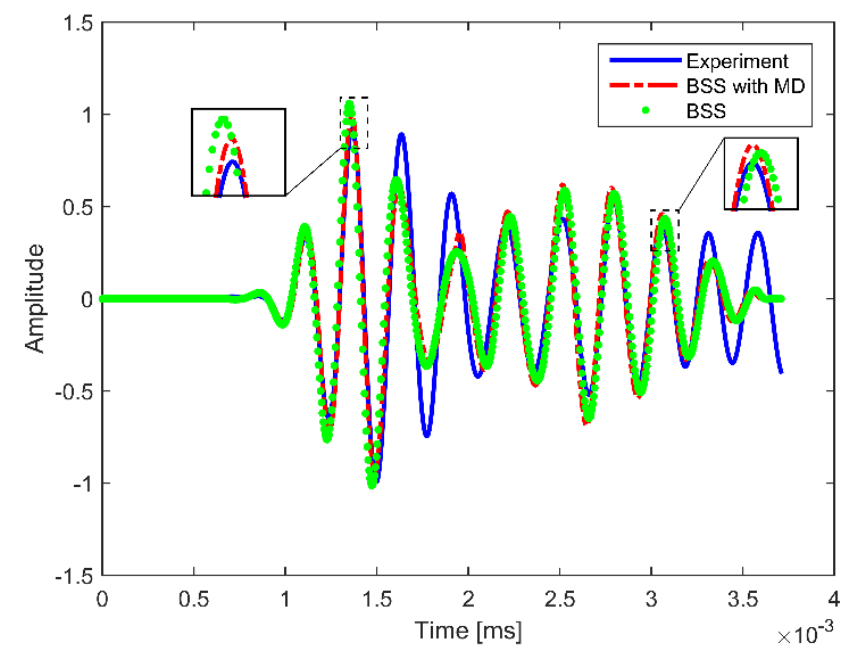

(e)

Figure 9. Comparison between the experimental measurements and the results obtained using BSS and BSS with MD in the time domain at (a) $15^{\circ} \mathrm{C}$ (b) $5^{\circ} \mathrm{C}$ (c) $-5^{\circ} \mathrm{C}$ (d) $-15^{\circ} \mathrm{C}$ (e) $-25^{\circ} \mathrm{C}$.

As illustrated, both methods give reasonable results compared to the experimental measurements at smaller temperatures intervals, however, in larger temperature intervals, the modified BSS method shows slightly better results. For deeper understanding the route mean square (RMS) of the error between the experimental results and each of the methods are calculated and illustrated in Fig. 10. The comparison shows that the BSS method with MD shows slightly better results for larger temperature intervals. Since the guided wave is a dispersive wave and there will be more mode separation at larger distances, it is expected that the modification of the BSS method using two stretch factors will give larger improvement when the guided wave travels longer inside the material. 


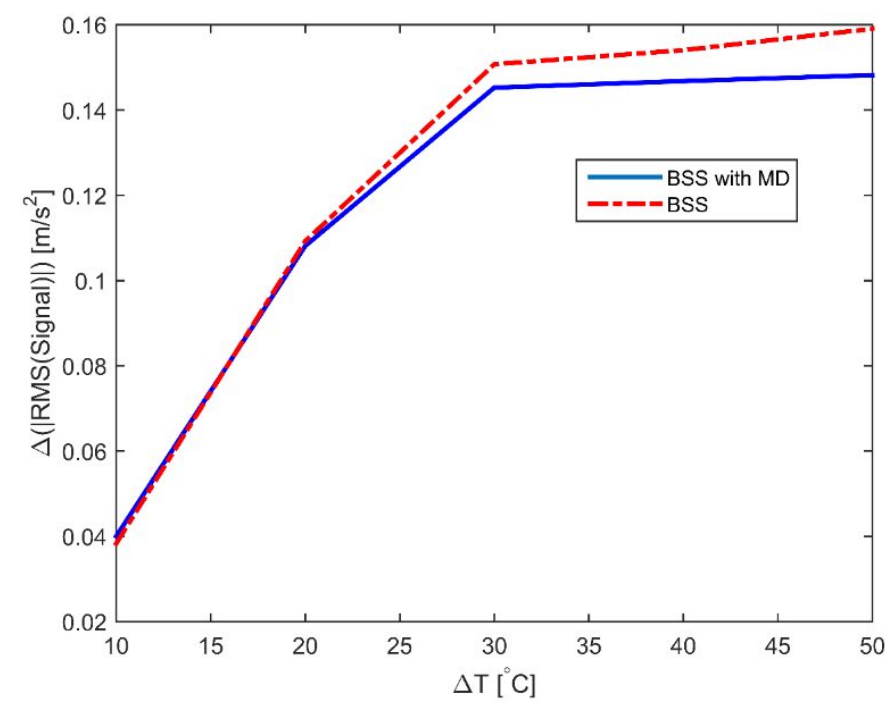

Figure 10. Comparison between the RMS error using the BSS and the BSS with MD for different temperature intervals.

\section{CONCLUSION AND OUTLOOK}

An experimental study is performed in order to understand the effects of temperature variations on guided waves propagating through a composite plate. Measurements show that due to the significant effect of temperature variation on the piezoelectric materials used in the transducer and the sensors, the amplitude of the signal decreases by lowering the temperature. Moreover, the existence of time lag in the received signal and its changes due to lowering the temperature prove that the group velocity of the wave increases within the temperature change. The orthotropic characteristics of the composite materials cause the rate of change of the amplitude and time lag to be different for symmetric and asymmetric modes. The rate of change of the amplitude is 1.75 and time lag is 5 times faster for asymmetric modes compared to symmetric modes when the temperature variation increases from $10^{\circ} \mathrm{C}$ to $50^{\circ} \mathrm{C}$.

In order to predict the signal at lower temperatures using the time signal at $25^{\circ} \mathrm{C}$, the BSS method has been modified and applied using different stretch factors for symmetric and asymmetric modes. The method is applied to the experimental data and compared with the BSS method with one stretch factor. Results show that BSS with MD gives slightly better results. The improvement is larger for larger temperature variations.

BSS with MD is a simple method with reasonable accuracy to handle the effects of temperature on the received signal from the propagation of guided waves inside composite structures. This method can be implemented for numerical simulations of wave propagation when temperature variations should be considered. Wind turbines are one of the major examples of engineering systems with composite structures which work under cold conditions. A numerical model can be built in order to simulate the propagation of guided waves in wind turbine blades to detect the accumulated ice on them using BSS with MD.

\section{ACKNOWLEDGEMENTS}

This project is part of a PhD project "Ice detection for smart de-icing of wind turbines" and it funded by Swedish Energy Agency. The authors would like to thank Mr. Jan Möller for all the technical support in the experimental tests. 


\section{REFERENCES}

[1] Lu, Y. and J.E. Michaels, "A methodology for structural health monitoring with diffuse ultrasonic waves in the presence of temperature variations." Ultrasonics. 43(9), p. 717-731 (2005).

[2] Shoja, S., V. Berbyuk, and A. Boström. "Investigating the application of guided wave propagation for ice detection on composite materials." Proc. International Conference on Engineering Vibration, Ljubljana, p. 152-161 (2015).

[3] Mazzeranghi, A. and D. Vangi, "Methodology for minimizing effects of temperature in monitoring with the acoustoultrasonic technique." Experimental Mechanics, 39(2), p. 86-91 (1999).

[4] Croxford, A.J., Wilcox, P.D., Drinkwater, B.W., and Konstantinidis, G., "Strategies for guided-wave structural health monitoring." Proc. of the Royal Society of London A: Mathematical, Physical and Engineering Sciences, 463(2087), p. 2961-2981 (2007).

[5] Croxford, A.J., Wilcox, P.D., Konstantinidis, G., and Drinkwater, B.W., "Strategies for overcoming the effect of temperature on guided wave structural health monitoring." (2007).

[6] Croxford, A.J., Moll, J., Wilcox, P.D., and Michaels, J.E. , "Efficient temperature compensation strategies for guided wave structural health monitoring." Ultrasonics. 50(4-5), p. 517-528 (2010).

[7] Dan, C.A., P. Kudela, and W. Ostachowicz. "Compensation of Temperature Effects on Guided Wave Based Structural Health Monitoring Systems." in EWSHM-7th European Workshop on Structural Health Monitoring. (2014).

[8] Sefrani, Y. and J.-M. Berthelot, "Temperature effect on the damping properties of unidirectional glass fibre composites." Composites Part B: Engineering. 37(4-5), p. 346-355 (2006).

[9] Salamone, S., Bartoli, I., Lanza Di Scalea, F., and Coccia, S., "Guided-wave Health Monitoring of Aircraft Composite Panels under Changing Temperature." Journal of Intelligent Material Systems and Structures. 20(9), p. 1079-1090 (2009). [10] Putkis, O., R.P. Dalton, and A.J. Croxford, "The influence of temperature variations on ultrasonic guided waves in anisotropic CFRP plates." Ultrasonics. 60, p. 109-116 (2015).

[11] Park, C.Y. and S.M. Jun, "Guided Wave Damage Detection in Composite Plates under Temperature Variations." Materials and Manufacturing Processes. 25(4), p. 227-231 (2010).

[12] Berbyuk, V., "Towards dynamics of controlled multibody systems with magnetostrictive transducers." Multibody System Dynamics. 18(2), p. 203-216 (2007).

[13] Lowe, M.J.S., D.N. Alleyne, and P. Cawley, "Defect detection in pipes using guided waves." Ultrasonics. 36(1-5), p. 147-154 (1998).

[14] Lanza di Scalea, F. and S. Salamone, "Temperature effects in ultrasonic Lamb wave structural health monitoring systems." The Journal of the Acoustical Society of America. 124(1), p. 161-174 (2008).

[15] Azaria, M. and D. Hertz, "Time delay estimation by generalized cross correlation methods." Acoustics, Speech and Signal Processing, IEEE Transactions on. 32(2), p. 280-285 (1984).

[16] Park, I., Y. Jun, and U. Lee, "Lamb wave mode decomposition for structural health monitoring." Wave Motion. 51(2), p. 335-347 (2014). 